\title{
In-situ Synthesis of Mixed Vanadium (IV and V) Oxides/Reduced Graphene Oxide Using Centella asiatica Extract
}

\author{
Sadhna Rai1 ${ }^{1}$ Rabina Bhujel ${ }^{1}$, Amrita Gupta ${ }^{2}$, Bibhu Prasad Swain ${ }^{3}$, Joydeep Biswas ${ }^{2, *}$ \\ ${ }^{1}$ Centre for Materials Science and Nanotechnology, Sikkim Manipal Institute of Technology, Sikkim Manipal \\ University, Majhitar, East Sikkim, 737136, India \\ 2 Department of Chemistry, Sikkim Manipal Institute of Technology, Sikkim Manipal University, Majhitar, \\ East Sikkim, 737136, India \\ ${ }^{3}$ Department of Physics, National Institute of Technology, Manipur, Langol-795004, Manipur, India
}

(Received 08 January 2021; revised manuscript received 12 February 2021; published online 25 February 2021)

Mixed vanadium (IV and V) oxides/reduced graphene oxide $\left(\mathrm{VO}_{2} / \mathrm{rGO}\right.$ and $\left.\mathrm{V}_{2} \mathrm{O}_{5} / \mathrm{rGO}\right)$ composite was synthesized by a green method. The green method prevents the use of hazardous chemicals, viz., hydrazine hydrate, which is commonly utilized for reducing the oxygen functionalities of graphene oxide (GO). The reduction of GO and incorporation of $\mathrm{VO}_{2}$ and $\mathrm{V}_{2} \mathrm{O}_{5}$ were performed simultaneously in a single concerted step using Centella asiatica extract. The composite was then characterized using UV-visible spectroscopy, X-ray Diffraction (XRD), Raman spectroscopy and scanning electron microscopy (SEM). This work shows the potential of Centella asiatica extract as a reducing agent.

Keywords: Vanadium oxide, Reduced graphene oxide, Centella asiatica, Green method, Reducing agent.

DOI: 10.21272/jnep.13(1).01031

\section{INTRODUCTION}

The research on nanoparticles has been intensified due to its dynamic and versatile applications. Scientific research on nanoparticles is trending today as they have many applications in the field of medicine, agriculture, physics, optics, electronics, cosmetics and many more. The remarkable properties of graphene led to its tremendous use in these fields [1-3]. Further, the composite of graphene other functionalities shows exciting properties due to interaction between them. Different approaches are adopted for the preparation of graphene, viz., epitaxial growth, chemical exfoliation, chemical vapor deposition, thermal reduction, chemical reduction and photoreduction [4-8]. However, the chemical reduction method being simple and time-efficient has become distinctly famous for it has the advantage of large production at a minimum cost. However, this method possesses a drawback as it utilizes hazardous chemicals, viz., hydrazine hydrate. Hence, Green synthesis is required to minimize the production of by-products, utilization of fewer resources, reduce the production and use of toxic chemicals. Among the available green methods, utilization of plant extract is seen to be the best and easy way to produce a large amount of metal/reduced graphene oxide composite. Bo et al. synthesized reduced graphene oxide using caffeic acid. This rGO was then used for potential application in sensing and energy storage [9]. Weng et al. prepared rGO/iron nanoparticles and used it for the removal of methylene blue [10]. Kadiyala et al. studied the potential of Syzygium cumini seed extract for preparation of gold nanoparticle decorated rGO and evaluated its biological applications [11]. Ramanathan et al. investigated the photocatalytic degradation of Rhodamine $\mathrm{B}$ with the help of $\mathrm{ZnO} / \mathrm{rGO}$ which nanocomposite was synthesized using grape fruit and Eichhornia crassipes leaf extract.
PACS numbers: 62.23.Kn, 62.23.Pq, 68.37.Hk, 87.64.Bx, 87.64.kp, 07.60.Rd
In the present work, the authors have evaluated the potential of Centella asiatica leaf extract for the onestep synthesis of mixed vanadium (IV and V) oxides/ rGO composite. Centella asiatica is a perennial herb found in India, Sri Lanka, Madagascar, South Africa, Australia, China, and Japan [12]. It has medicinal values and contains antioxidants. It contains the following types of chemical compounds viz. triterpenoids, volatile and fatty acids, alkaloids, glyceroids, flavonoids, and other compounds such as vitamin B, C and some amino acids [13]. It is easily found in the marshy lands and is nontoxic. Hence, in the present study, its reducing ability was evaluated.

\section{EXPERIMENTAL DETAILS}

\subsection{Materials}

Graphite powder (Loba Chemie), sodium nitrate $\left(\mathrm{NaNO}_{3}\right)$ (Rankem), sulphuric acid $\left(\mathrm{H}_{2} \mathrm{SO}_{4}\right)$ (Rankem), potassium permanganate $\left(\mathrm{KMnO}_{4}\right)$ (Rankem), hydrogen peroxide $\left(\mathrm{H}_{2} \mathrm{O}_{2}\right)$ (Rankem), hydrochloric acid $(\mathrm{HCl})$ (SD Fine Chem Ltd), vanadium pentoxide $\left(\mathrm{V}_{2} \mathrm{O}_{5}\right)$ (Merck) and ammonium hydroxide $\left(\mathrm{NH}_{4} \mathrm{OH}\right)$ (Merck) were of analytical grade purity. The leaves of Centella asiatica were obtained from Soreng, West Sikkim.

\subsection{Synthesis}

The Centella asiatica leaves were washed and dried. The dried leaves were crushed using a mortar pestle. $5 \mathrm{~g}$ of this ground leaves were taken in $100 \mathrm{ml}$ DI water and refluxed for $1 \mathrm{~h}$. The extract was filtered and cooled and stored in the refrigerator for further use. $0.1 \mathrm{~g}$ of GO synthesized using conventional Hummer method was dispersed in $50 \mathrm{ml}$ DI water [14]. $1 \mathrm{mM}(20 \mathrm{ml})$ vanadium pentoxide $\left(\mathrm{V}_{2} \mathrm{O}_{5}\right)$ in ammonium hydroxide

\footnotetext{
* joydeep.biswas@smit.smu.edu.in

The results were presented at the International Conference on Multifunctional Nanomaterials (ICMN2020)
} 
$\left(\mathrm{NH}_{4} \mathrm{OH}\right)$ was added dropwise to the dispersion. $10 \mathrm{ml}$ of the above extract was poured into the mixture and stirred. It was then refluxed for $6 \mathrm{~h}$ at $90{ }^{\circ} \mathrm{C}$. A black product was obtained after $6 \mathrm{~h}$ which was then filtered, washed several times with DI water and dried. The sample, as mentioned above, was labelled as V10. The volume of extract was varied and two more samples were prepared. The samples were labelled as V20 and V30 for 20 and $30 \mathrm{ml}$ of extract, respectively.

\subsection{Characterization Techniques}

The UV-visible spectra were collected in UV-1800 SHIMADZU UV spectrophotometer. SEM micrographs were obtained from ZEISS EVO 60 scanning electron microscope. The XRD patterns and Raman spectra were obtained from Bruker D8 Advance X-ray diffractometer and Jobin Yvon Horiba LABRAM HR-800 Visible micro-Raman spectrometer, respectively.

\section{RESULTS AND DISCUSSION}

\subsection{Morphological Characterization}

The morphological study of the prepared samples was done using SEM micrographs. Fig. 1 shows highly folded and agglomerated sheets of GO. However, the agglomeration is reduced as GO is reduced to rGO in V30 as can be seen in Fig. 1b. The agglomeration is removed because the oxygen functional groups are removed as GO is reduced to rGO. Also, it is visible that vanadium oxide particles are anchored on rGO sheets. From SEM it is clearly seen that the Centella asiatica extract can reduce the GO to rGO and simultaneously form vanadium oxide nanoparticles.

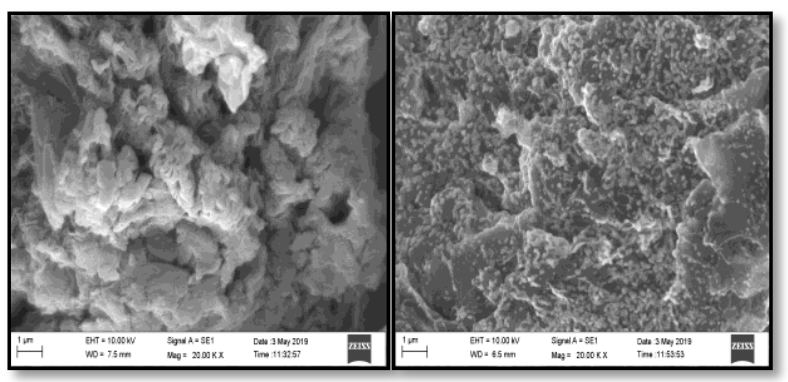

Fig. 1 - SEM images of GO and V30

\subsection{Structural Characterization}

XRD pattern of GO shows an intense peak at $2 \theta=10^{\circ}$, which corresponds to the (002) plane (Fig. 2a) [15]. This peak disappeared in the XRD patterns of mixed vanadium (IV and V) oxides/reduced graphene oxide $\left(\mathrm{VO}_{2} / \mathrm{rGO}\right.$ and $\left.\mathrm{V}_{2} \mathrm{O}_{5} / \mathrm{rGO}\right)$ composite, which suggests that GO is reduced to rGO [16]. However, the XRD patterns of mixed vanadium (IV and V) oxides/ reduced graphene oxide $\left(\mathrm{VO}_{2} / \mathrm{rGO}\right.$ and $\left.\mathrm{V}_{2} \mathrm{O}_{5} / \mathrm{rGO}\right)$ composite show peaks centered at $2 \theta$ values of $23.2^{\circ}, 29.4$, $39.4^{\circ}, 43.3^{\circ}, 47.6^{\circ}, 48.4^{\circ}$, corresponding to (201), (002), (112), (003), (203), (020) planes of $\mathrm{VO}_{2}$. Additionally, the peaks belonging to $\mathrm{V}_{2} \mathrm{O}_{5}$ are observed at $2 \theta$ values of 13.3 and $35.9^{\circ}$, which are attributed to (002) and (111) planes, respectively [17]. The result, as mentioned above, shows that in the composite, vanadium exists in +5 and +4 oxidation states. As the extract amount has increased, the intensity of peak at $13.3^{\circ}$ for $\mathrm{V}_{2} \mathrm{O}_{5}$ is found to be diminished where the intensity of peak at $29.4^{\circ}$ for $\mathrm{VO}_{2}$ is found to be enhanced. However, such changes are not observed for the samples, viz., V20 and V30.

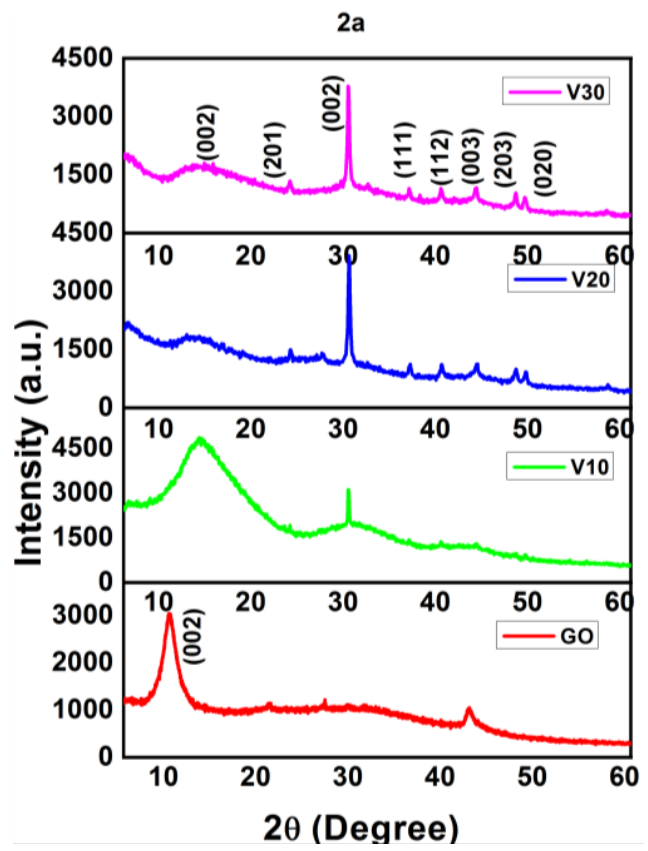

2b

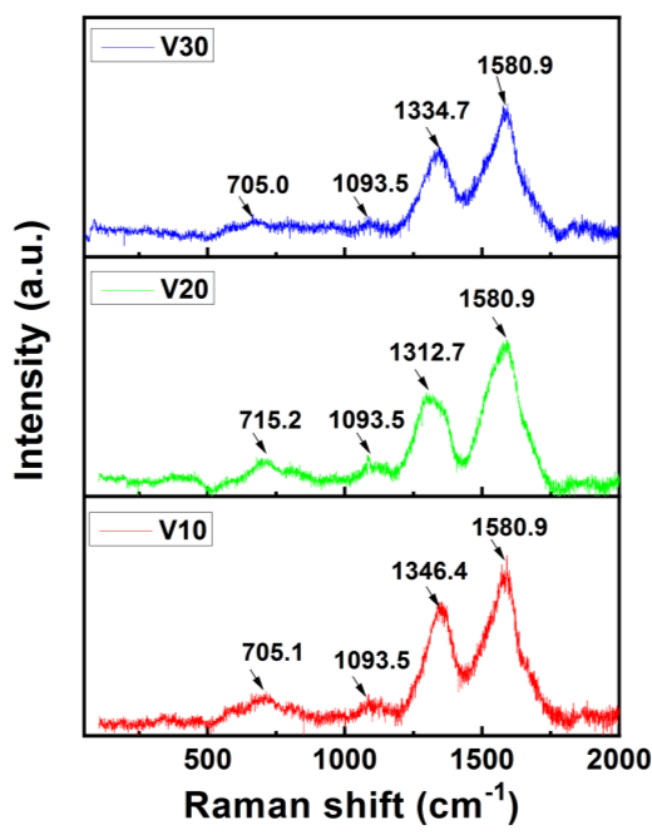

Fig. 2 - (a) X-ray diffraction of GO, V10, V20 and V30 and (b) Raman spectra of V10, V20 and V30

The crystallite size of the $\mathrm{VO}_{2}$ nanoparticles was calculated using equation (1) considering the (002) plane

$$
D=K \lambda / \beta \cos \theta,
$$

where $D, K, \lambda, \beta$ and $\theta$ refer to the crystallite size, shape factor (0.9), wavelength of X-ray, full width half maximum and Bragg angle, respectively. 

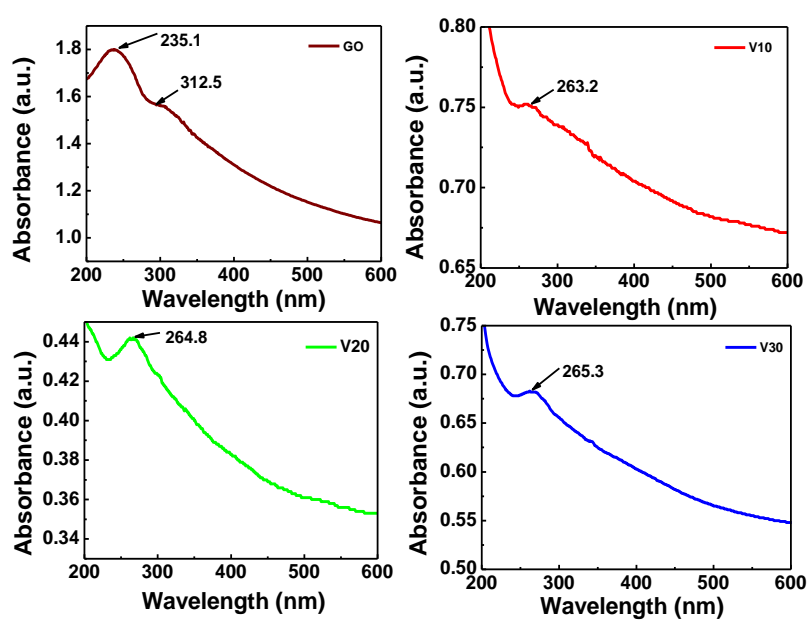

Fig. 3 - UV-visible spectra of GO, V10, V20 and V30

The crystallite size of $\mathrm{VO}_{2}$ nanoparticles in $\mathrm{V} 10$, V20 and V30 was found to be $42.9,34.3$ and $31.7 \mathrm{~nm}$, respectively.

The Raman spectra of V10, V20 and V30 are presented in Fig. 2b. The band at $\sim 700$ and $1000 \mathrm{~nm}$ are related to stretching modes of $\mathrm{V}-\mathrm{O}$ and $\mathrm{V}=\mathrm{O}$, respectively [17, 18]. The characteristic Raman band at $\sim 1300$ and $1500 \mathrm{~cm}^{-1}$ correspond to D and G bands for carbonaceous materials [19]. The D band refers to defect states while the $\mathrm{G}$ band refers to $s p^{2}$ domains of carbon compounds [20]. The $I_{D} / I_{G}$ was calculated as $0.58,0.43$ and 0.45 for V10, V20 and V30, respectively.

\subsection{Optical Characterization}

The UV-visible spectra of GO, V10, V20 and V30 are given in Fig. 3. There are two prominent peaks located at $235.1 \mathrm{~nm}$ and $312.5 \mathrm{~nm}$. The absorption peak

\section{REFERENCES}

1. C.P.P. Wong, C.W. Lai, K.M. Lee, S.B. Abd Hamid, Materials (Basel).8, 7118 (2015).

2. S.C. Ray, Applications of Graphene and Graphene-Oxide Based Nanomaterials (Elsevier: 2015).

3. S. Ni, F. Han, W. Wang, D. Han, Y. Bao, D. Han, H. Wang, L. Niu, Sensor. Actuat. B: Chem. 259, 963 (2018).

4. M. Yi, Z. Shen, J. Mater. Chem. A. 3, 11700 (2015).

5. X. Gao, J. Jang, S. Nagase, J. Phys. Chem. C 114, 832 (2010).

6. Y. Matsumoto, M. Koinuma, S.Y. Kim, Y. Watanabe, T. Taniguchi, K. Hatakeyama, H. Tateishi, S. Ida, ACS Appl. Mater. Interface. 2, 3461 (2010).

7. W. Yang, G. Chen, Z. Shi, C.-C. Liu, L. Zhang, G. Xie, M. Cheng, D. Wang, R. Yang, D. Shi, K Watanabe, T. Taniguchi, Y. Yao, Y. Zhang, G. Zhang, Nat. Mater. 12, 792 (2013).

8. C.K. Chua, M. Pumera, Chem. Soc. Rev. 43, 291 (2014).

9. Z. Bo, X. Shuai, S. Mao, H. Yang, J. Qian, J. Chen, J. Yan, K. Cen, Sci. Rep. 4, 4684 (2015)

10. X. Weng, Z. Lin, X. Xiao, C. Li, Z. Chen, J. Clean. Prod. 203, 22 (2018).

11. N.K. Kadiyala, B.K. Mandal, S. Ranjan, N. Dasgupta, Mater. Sci. Eng. C 93, 191 (2018). at $235.1 \mathrm{~nm}$ is for the excitation of an electron from $\pi \rightarrow \pi^{*}$ in $\mathrm{C}=\mathrm{C}$ of the aromatic ring and that at $312.5 \mathrm{~nm}$ is ascribed to $n \rightarrow \pi^{*}$ electronic transition of $\mathrm{C}=\mathrm{O}$ group [21]. Sharp peaks are centered at 263.2, 264.8 and $265.3 \mathrm{~nm}$ for V10, V20 and V30, respectively, which is for the $\pi \rightarrow \pi^{*}$ electronic transitions. Furthermore, the peak for the electronic transition of $n \rightarrow \pi^{*}$ is absent in the composite because the $\mathrm{C}=\mathrm{O}$ bonds are reduced by Centella asiatica extract [22]. Thus, from UVvisible spectroscopy study also, it is found that Centella asiatica extract can be used as a reducing agent.

\section{CONCLUSIONS}

A green approach is adopted for the synthesis of mixed $\mathrm{VO}_{2} / \mathrm{rGO}$ and $\mathrm{V}_{2} \mathrm{O}_{5} / \mathrm{rGO}$ composite. Here, we used Centella asiatica, which is a locally available plant. This study shows that Centella asiatica extract has the potential of a reducing agent. From UV-Visible, XRD and Raman data analysis, it is found that Centella asiatica extract can successfully reduce GO. Thus the use of hazardous chemicals, viz., hydrazine hydrate is prevented, which can harm human health as well as the environment. Furthermore, the in-situ synthesis of mixed $\mathrm{VO}_{2} / \mathrm{rGO}$ and $\mathrm{V}_{2} \mathrm{O}_{5} / \mathrm{rGO}$ composite can save time as well as resources.

\section{ACKNOWLEDGEMENTS}

The authors thank UGC-DAE Consortium for Scientific Research, Indore, India (Project Ref: CSR-ICMSR-07/CRS-215/2017-18/1296) for financial support. The authors are also grateful towards Dr. Mukul Gupta and Mr. Layanta Behera; and Dr. Vasanth Sathe and Mr. Ajay Rathore from UGCDAE Consortium for Scientific Research, Indore, India for providing XRD and Raman data, respectively.

12. P.K. Mukherjee, N. Maity, N.K. Nema, B.K. Sarkar, Phytomedicine 19, 64 (2011).

13. B.S. Siddiqui, H. Aslam, S.T. Ali, S. Khan, S. Begum, J. Asian Nat. Prod. Res. 9, 407 (2007).

14. R. Bhujel, S. Rai, U. Deka, B.P. Swain, J. Alloy. Compd. 792, 250 (2019).

15. A.A. Boochakravarthy, M. Dhanasekar, S.V. Bhat, AIP Adv. 10, 085127 (2020).

16. S. Rai, R. Bhujel, J. Biswas, B.P. Swain, Ceram. Int. 45, 14136 (2019).

17. S. Kundu, B. Satpati, T. Kar, S.K. Pradhan, J. Hazard. Mater. 339, 161 (2017).

18. H.K. Koduru, H.M. Obili, G. Cecilia, Int. Nano Lett. 3, 24 (2013).

19. X. Yan, Y. Li, M. Li, Y. Jin, F. Du, G. Chen, Y. Wei, J. Mater. Chem. A 3, 4187 (2015).

20. R. Bhujel, S. Rai, B.P. Swain, Appl. Nanosci. 9, 1319 (2019).

21. Y. Yang, T. Liu, Appl. Surf. Sci. 257, 8950 (2011).

22. S. Kundu, B. Satpati, M. Mukherjee, T. Kar, S.K. Pradhan, New J. Chem. 41, 3634 (2017). 